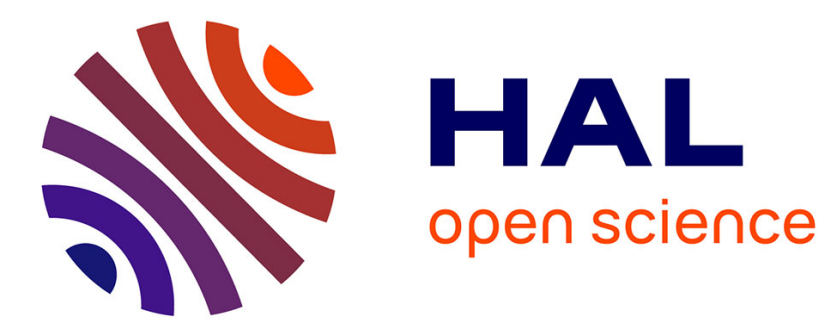

\title{
L'exil dans les Intercenales de Leon Battista Alberti
}

Donatella Bisconti

\section{To cite this version:}

Donatella Bisconti. L'exil dans les Intercenales de Leon Battista Alberti. Arzanà. Cahiers de littérature médiévale italienne, 2015, 16-17, pp.403 - 417. 10.4000/arzana.234 . hal-01898845

\section{HAL Id: hal-01898845 \\ https://hal.science/hal-01898845}

Submitted on 18 Oct 2018

HAL is a multi-disciplinary open access archive for the deposit and dissemination of scientific research documents, whether they are published or not. The documents may come from teaching and research institutions in France or abroad, or from public or private research centers.
L'archive ouverte pluridisciplinaire HAL, est destinée au dépôt et à la diffusion de documents scientifiques de niveau recherche, publiés ou non, émanant des établissements d'enseignement et de recherche français ou étrangers, des laboratoires publics ou privés. 


\section{Arzanà}

Cahiers de littérature médiévale italienne

16-17 | 2013

Écritures de l'exil dans l'Italie médiévale

\section{L'exil dans les Intercenales de Leon Battista Alberti}

Ou de l'autonomie politique et intellectuelle du citoyen

L'esilio nelle Intercenales di Leon Battista Alberti. O dell'autonomia politica e intellettuale del cittadino

\section{Donatella Bisconti}

\section{revues.org}

Édition électronique

URL : http://arzana.revues.org/234

DOI : 10.4000/arzana.234

ISSN : 2429-9499

\section{Éditeur \\ Presses Sorbonne Nouvelle}

\section{Édition imprimée}

Date de publication : 1 avril 2013

Pagination : 403-417

ISBN : $978-2-87854-583-8$

ISSN : 1243-3616

Ce document vous est offert par Centre national de la recherche scientifique (CNRS)

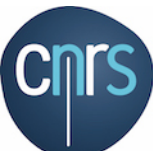

Référence électronique

Donatella Bisconti, "L'exil dans les Intercenales de Leon Battista Alberti », Arzanà [En ligne],

16-17 | 2013, mis en ligne le 16 juin 2015, consulté le 06 avril 2017. URL : http://arzana.revues.org/234

; DOI : 10.4000/arzana.234 


\section{L'exil dans les Intercenales de Leon Battista Alberti : ou de l'autonomie politique et intellectuelle du citoyen}

ans son essai sur l'humorisme ${ }^{1}$, Pirandello, en distinguant l'humo-
risme d'autres formes de comique, affirme:

L'umorismo [...] per il suo intimo, specioso, essenziale processo, inevitabilmente scompone, disordina, discorda; quando, comunemente, l'arte in genere, com'era insegnata dalla scuola, dalla retorica, era sopra tutto composizione esteriore, accordo logicamente ordinato ${ }^{2}$.

En effet, selon l'écrivain sicilien, l'humorisme n'est ni la parodie, qui déforme et exagère, ni l'ironie, quella tal contradizione fittizia tra quel che si dice e quel che si vuole sia inteso (" cette contradiction fictive entre ce qui est dit et ce que l'on veut faire entendre ").

Lorsque Pirandello passe en revue les humoristes italiens, il lui vient d'abord à l'esprit Machiavelli, et tout de suite après Giordano Bruno, qui se définissait « in tristitia hilaris, in hilaritate tristis ». En réfléchissant sur le Spaccio della bestia trionfante, publié en 1584, il souligne l'humorisme amer d'un auteur qui, pour démontrer que la divinité s'intéresse à l'homme aussi bien dans les grandes que dans les petites choses, nous livre par le menu la liste des consignes de Jupiter concernant la récolte des fruits, la naissance et le destin de deux-cent-cinquante cafards, le moment précis où une femme tombera enceinte. Cette description minutieuse a comme effet prévisible de nous faire douter de la providence d'un Dieu occupé à compter les cafards et à décider du sort de chaque melon de notre jardin.

1. Nous adoptons cette traduction qui sauvegarde la portée théorique de l'umorismo selon Pirandello.

2. Pirandello, Luigi, L'umorismo, in L'umorismo e altri saggi, Firenze, Giunti, 1994, p. 37 : «L'humorisme, par son procédé intime, spécifique, essentiel, inévitablement décompose, désordonne, scinde, tandis que l'art en général, tel qu’il était enseigné par l'école, par la rhétorique, était surtout une composition extérieure, un accord ordonné logiquement. » 
Mais plus d'un siècle et demi auparavant, ce Dieu lointain, impersonnel et peu soucieux de l'humanité, n'en déplaise à l'Église, est déjà présent chez L. B. Alberti, lorsque, dans l'Intercenalis Religio, il se moque de la confiance des hommes dans les prières qu'ils élèvent à la divinité, dont ils espèrent être exaucés mais dont ils ne saisissent pas le statut contradictoire et l'intime égoïsme. Les dévots, fait remarquer Libripeta à Lepidus, en voulant soumettre les dieux à leurs demandes, font d'eux des pillards, qui élargissent à l'un ce qu'ils soustraient à l'autre. Lepidus répond qu'il veut faire des dieux non pas des voleurs mais des ouvriers sachant faire pousser des choux en or dans son potager, puis demande à un Libripeta scandalisé s'il pourrait nier que les dieux viennent en aide à l'homme dans les adversités. Libripeta démontre alors que les maux qui affligent l'humanité n'ont pas d'autre cause que les hommes euxmêmes; si par contre les responsables en étaient les dieux, ce ne seront pas nos prières qui les détourneront de leurs décisions. D’ailleurs, comment les dieux auraient-ils le temps de penser aux menus détails de nos petites vies, occupés qu'ils sont à diriger le cours des astres, à réguler le climat et les autres phénomènes naturels? Et si vraiment ils désirent se pencher sur des objets de moindre importance, ils écouteront plus volontiers le chant des grillons et des cigales que les requêtes vaines d'hommes scélérats, car les méchants seuls, qui demandent sans mesure et ne supportent pas leurs malheurs, s'adressent à la divinité, tandis que les bons sont pleinement satisfaits de ce qu'ils possèdent et ne succombent jamais à l'adversité ${ }^{3}$.

Selon un procédé qu'Alberti affine tout au long des Intercenales, les rôles respectifs des interlocuteurs, loin d'être figés, sont, comme ici, inversés. En effet, Lepidus, l'auteur fictif de la Philodoxeos fabula, est dans plusieurs intermèdes l'un des alter ego de Battista, qui se qualifie comme écrivain "facetus " et compare ses Intercenales aux médicaments administrés par son ami Paolo Toscanelli : si les médicaments soignent le corps, les Intercenales soignent l'esprit de ses malheurs par le rire. Libripeta, de son côté, est en général l'antagoniste de Lepidus. Son sobriquet, sous lequel se cache l'humaniste Niccolò Niccoli, fait allusion à sa manie de bibliophile, stérile par ailleurs, car Niccoli n'écrivit aucune ouvre, et à sa vénération immodérée pour l'Antiquité. Sa bizarrerie, qui est empruntée à la fois au caractère de Niccoli et à l'attitude des philosophes cyniques, dont Alberti critique l'excentricité, l'excès et une certaine immoralité, est en même temps l'expédient qui permet à Libripeta sa liberté de parole, parfois simple médisance sur fond de vérité, parfois vérité incommode proférée par un personnage peu crédible.

3. Alberti, Leon Battista, Religio, in Intercenales, a cura di Franco Bacchelli e Luca d'Ascia, premessa di Alberto Tenenti, Bologna, Pendragon, 2003, livre I, p. 22-31. 
Ainsi, dans Religio, la position laïque et anti-providentielle d'Alberti n'est pas affirmée nettement, car Lepidus refuse de suivre son interlocuteur sur ce terrain, mais le germe du doute est semé. Ce procédé a également un autre avantage : les alter ego de Battista dans les Intercenales, Philoponius et Lepidus, ne sont pas entièrement ses porte-parole. Nous ne pouvons pas les identifier tout court avec Alberti, même lorsqu'ils font allusion aux vicissitudes de l'auteur ou de sa famille. Par ce biais, les éléments biographiques éparpillés dans les Intercenales, loin d'être un témoignage fiable, sont plutôt le point de départ de la discussion : le prétexte biographique replace ainsi un épisode isolé dans une dimension plus générale, voire universelle, et en tout cas partagée par toute l'humanité. En effet, il ne faut jamais oublier que la perception du "moi " n'est au Xv siècle pas la même que la nôtre. Leopardi affirmait que l'humour des Antiques était concret et corpulent, l'humour des modernes est devenu presque une ombre, de l'éther ${ }^{4}$; plus tard Pirandello dira que dans ce monde " orbo di dei ", dans le naufrage de l'existence, la science nous fournit une seule certitude, que la vie est un mal dépourvu de tout sens ${ }^{5}$, et l'humorisme apparaît alors comme une forme de représentation incontournable du mouvement incessant du monde extérieur et surtout du monde intérieur.

Ebranlé par le relativisme du $\mathrm{xx}^{\mathrm{e}}$ siècle, le " moi » se déstructure, mais au $\mathrm{XV}^{\mathrm{e}}$ siècle, au moment du réveil de l'Humanisme, la représentation du " moi » n'est pas intériorisée, la réflexion morale a besoin d'un interlocuteur extérieur, une sorte de double ou de miroir, posant les questions et faisant émerger le doute, la contradiction, la conscience de l'erreur. Alberti multiplie alors les personnages qui révèlent tour à tour l'une des innombrables facettes de la vie intérieure de l'homme ou des problèmes qui l'agitent. Ce choix, qui est certainement dicté par la prudence, lui évite aussi de prendre une position absolue et définitive : la discussion reste ouverte, et l'échange d'opinions toujours possible. D'un autre côté, le dédoublement du personnage permet le procédé humoristique et la représentation du conflit entre le sujet et le monde extérieur :

L'umorismo è un fenomeno di sdoppiamento nell'atto della concezione; è come un'erma bifronte, che ride per una faccia del pianto della faccia opposta. La riflessione diventa come un demonietto che smonta il congegno

4. Leopardi, Giacomo, Zibaldone di pensieri, in Tutte le opere, introduzione di Walter Binni, a cura di Walter Binni con la collaborazione di Enrico Ghidetti, vol. II, Firenze, Sansoni Editore, 1969, p. 41.

5. Pirandello, Luigi, Arte e coscienza d'oggi, in L'umorismo e altri saggi, op. cit., p. 237 sq. 


\section{DONATELLA BISCONTI}

dell'immagine, del fantoccio messo sú dal sentimento; lo smonta per veder come è fatto; scarica la molla, e tutto il congegno ne stride, convulso ${ }^{6}$.

Dans les Intercenales, cet échange est, d'une certaine façon, toujours « mis en scène ", sous la forme d'un dialogue, voire d'une scène de comédie, d'un apologue, d'une Ě $\kappa \varphi \rho \alpha \sigma i \varsigma$, d'une illustration d'emblèmes et de symboles. S'inspirant de modèles antiques, tels que Lucien de Samosate, notamment pour les dialogues, et Esope, pour les apologues, Alberti actualise leur discours en faisant allusion à la société contemporaine et en en déplaçant l'objectif : si Lucien, esprit essentiellement areligieux, vise par sa moquerie la vie future, la providence, l'idée même de la divinité, Alberti ne remet pas en question l'existence des dieux, mais essaie de concilier les deux pôles à l'intérieur desquels sa pensée oscille, entre un déterminisme inéluctable qui découle de la « Necessitas " stoïcienne, et donc d'un enchaînement de causes et d'effets auquel toute destinée est astreinte ${ }^{7}$, et la confiance dans les facultés intellectuelles et pragmatiques de l'homme. Si les fables d'Esope ont une finalité moralisante non ambiguë, les significations des apologues d'Alberti sont multiples et reflètent le conflit entre deux ou plusieurs points de vue.

Les Intercenales ayant trait à l'exil, que celui-ci constitue le sujet central du texte ou qu'on l'y évoque par moments, manifestent tout particulièrement cette multiplicité de points de vue. Tout d'abord, l'exil est une condition concrète, directement expérimentée par l'auteur. Fils d'exilé, né à Gênes, " apud exteras nationes [...] educatus ${ }^{8}$ ", Alberti découvre la ville natale de ses ancêtres, idéalisée dans les souvenirs de ses proches auparavant exilés, autour de 1430, après la réintégration des Alberti à Florence en 1428 : dans le même

6. Pirandello, Luigi, Arte e scienza, in L'umorismo e altri saggi, op. cit., p. 175 : «L'humorisme est un phénomène de dédoublement dans l'acte de la conception : il est comme un Hermès bifrons qui rit par un visage des pleurs du visage opposé. La réflexion devient une sorte de petit démon qui démonte le mécanisme de l'image, du pantin mu par le sentiment; il le démonte pour voir comment il est fait; il décharge le ressort, et tout le mécanisme grince, en proie à des mouvements convulsifs ".

7. Alberti, Leon Battista, Fatum et pater infelix, in Intercenales, op. cit., p. 536-549.

8. Fubini, Riccardo, Menci Gallorini, Anna, "L'autobiografia di L.B.A. Studio e edizione ", Rinascimento, n.s. XII, 1972, p. 70 : Patriam enim linguam, apud exteras nationes per diutinum familiae Albertorum exilium educatus, non tenebat, et durum erat hac in lingua scribere eleganter atque nitide, in qua tum primum scribere assuesceret. ("En effet, puisqu'il avait reçu son éducation à l'étranger, à cause de l'exil prolongé de sa famille, il ne pratiquait pas la langue de sa patrie, et il lui était difficile d'écrire dans cette langue, dès qu'il avait entrepris de le faire, avec élégance et propriété. »). 
laps de temps où l'obtention du prieuré de San Martino in Gangalandi, près de Florence, lui garantit une source de revenus qui, avec son emploi d'abréviateur (ensuite d'écrivain) apostolique, rendit plus confortable sa position économique et sociale 9 . Dans le De familia, lorsque l'exil des Alberti est évoqué, l'accent porte sur le malheur actuel s'opposant à l'ancien bonheur, si bien que Lionardo peut considérer comme heureux les jeunes Alberti morts dans leur patrie sans connaitre la dureté, les humiliations et les privations qui découlent d'un bannissement injuste ${ }^{10}$; en illustrant la diaspora des membres de la famille dans toute l'Europe et en Orient, il déplore la dissolution des liens d'amitié qui, à l'intérieur de Florence, garantissaient la transmission de la réputation des Alberti ${ }^{11}$. La volonté de rehausser le nom de la famille et d'en souligner les mérites, passés et actuels, explique en partie, bien que non totalement, l'activisme intellectuel de Battista dès son arrivée à Florence, sa productivité littéraire et scientifique, l'initiative du Certame coronario. D'un autre côté, Alberti est un exilé à l'intérieur de sa propre famille : enfant illégitime comme son frère Carlo, tardivement légitimé, exclu de l'héritage de son père Lorenzo, qui avait légué à lui-même et à Carlo une somme importante, mais disproportionnée par rapport à l'ampleur de son patrimoine, il ne put obtenir sa part d'héritage ni de Ricciardo, frère et exécuteur testamentaire de Lorenzo, ni de son fils Antonio, et dut entamer de longues procédures judiciaires contre Bernardo, l'enfant de ce dernier, pour se faire reconnaître la propriété de la moitié de la maison de son grand-père Benedetto, un contentieux devenu plus symbolique que substantiel au fil des années ${ }^{12}$.

9. Nous ne connaissons pas la date exacte à laquelle le bénéfice de San Martino in Gangalandi fut attribué à Alberti, mais nous pouvons établir un terminus a quo, puisque le précédent prieur mourut en 1430. Luca Boschetto, Leon Battista Alberti e Firenze. Biografia, storia, letteratura, Firenze, Olschki, 2000, p. 79 remarque que les deux seuls documents de la période romaine d'Alberti proviennent des archives florentines et portent les dates de 1432 et 1433 : le document de 1432 est une dispense accordée par Eugène IV pour la naissance illégitime d'Alberti et confirme l'attribution du bénéfice; le second, qui est une recommandation adressée par la Seigneurie florentine au cardinal Francesco Condulmer, émane de la Chancellerie florentine dont le chancelier était Leonardo Bruni, qui aida Alberti à se frayer un chemin dans le milieu difficile de la curie.

10. Alberti, Leon Battista, I libri della famiglia, in Opere volgari, a cura di Cecil Grayson, vol. I, I libri della famiglia, Cena familiaris, Villa, Bari, Laterza, 1960, 1. I, p. 38-39.

11. Ibid., 1. II, p. 85.

12. Pour le différend qui opposa Leon Battista Alberti à son cousin Bernardo di Antonio, cf. Paola Benigni, "Tra due testamenti, riflessioni sul alcuni aspetti problematici della bibliografia albertiana ", in La vita e il mondo di Leon Battista Alberti : atti 


\section{DONATELLA BISCONTI}

D'autre part, si Antonio ne versa pas la somme due, c'est aussi à cause de la catastrophe financière qui frappa les compagnies Alberti de Ponente ${ }^{13}$ et de Rome. La responsabilité de ce désastre doit être attribuée surtout à la mauvaise gestion et à l'incompétence d'Antonio di Ricciardo et de Benedetto di Bernardo, cousins de Battista. Benedetto prit la fuite en août 1436, après la banqueroute, et Antonio fut arrêté, puis banni en février 1439. La faillite des compagnies de Ponente entraîna à son tour le krach de la compagnie de Rome, victime en quelque sorte innocente du système financier des Alberti. C'est à partir de ce moment-là qu'on assiste au déclin des Alberti non seulement sur le plan économique, mais également politique : une branche des Alberti participe toujours à la politique (les frères Giannozzo et Antonio di Tommaso qui maintiennent " onore » et " sostanze »), mais n'est plus au centre du pouvoir ${ }^{14}$. Dans ces conditions, il était difficile, pour Battista, de revendiquer l'honnêteté et la loyauté de sa famille. L'exil, qui avait été utilisé comme une arme politique contre les Alberti entre la fin du XIv et le début du Xve siècles, est maintenant décrété comme une mesure infamante contre Antonio, et cette tache d'infamie ne peut que se répandre sur les autres membres de la famille. L'exil a donc tout d'abord une dimension objective dans les Intercenales, dont témoignent surtout Pupillus, Erumna et, en partie, Divitie.

La représentation de Philoponius, "l'ami des épreuves », dans Pupillus, est le portrait d'un exclu, placé sous la tutelle de ses proches, mais en même temps rejeté par ceux-ci et privé de ressources :

Nam is, relictus puer, patre defuncto, sine ullis parentibus, proscriptus a patria coniunctissimisque ab affinibus non modo bonis omnibus paternis spoliatus, sed etiam a suorum domestica familiaritate et convictu exclusus atque omnino ita abiectus fuit, ut apud extraneos sibi esset mendicandum. Erat istiusmodi fortuna adolescenti gravis quidem, sed illud longe gravius, quod impiissimi adolescentis affines summopere elaborabant, ne adolescens Philoponius in

dei convegni internazionali del Comitato nazionale VI centenario della nascita di Leon Battista Alberti, Genova, 19-21 febbraio 2004, Firenze, L. S. Olschki, 2008, p. 173-189.

13. Il s'agit de la holding qui œuvrait en Europe occidentale et avait des filières à Londres, Bruges et Cologne. Pour son fonctionnement, voir Luca Boschetto, Leon Battista Alberti e Firenze, op. cit., p. 21 sq.

14. Pour les vicissitudes financières et le déclin politique des Alberti à partir de 1436, voir Luca Boschetto, Leon Battista Alberti e Firenze, op. cit., p. 39-67. 
his studiis litterarum posset prosequi, quibus non mediocri cum expectatione civium et litteratorum omnium admiratione proficiebat ${ }^{15}$.

Par son excès même, ce portrait d'une victime doit nous mettre en garde contre toute tentation d'interprétation autobiographique : Alberti, qui n'était pas dans une condition d'indigence au moment de la rédaction des Intercenales grâce à son emploi à la curie et au bénéfice de San Martino, fut en effet privé de l'héritage par son père lui-même, pas par ses proches, et ne fut pas exclu de la fréquentation du cercle familial. Ses relations se dégradèrent avec Benedetto et Antonio, mais non pas avec d'autres Alberti, comme Francesco d'Altobianco. Le conflit au sujet des études doit être ramené à sa juste dimension, car Alberti lui-même nous explique dans la Vita latina sa décision d'abandonner temporairement les études de droit au profit des sciences en raison d'une dépression nerveuse ${ }^{16}$. Le penchant pour les lettres, contrarié par les proches de Philoponius, est plutôt à mettre en relation avec le discours de Lionardo, dans le De familia, sur le devoir des pères d'encourager les qualités naturelles de leurs enfants, surtout lorsqu'ils sont aptes aux arts, aux sciences ou à la gloire

15. Alberti, Leon Battista, Pupillus, in Intercenales, op. cit., livre I, p. 12 : «En effet celui-ci, laissé orphelin par son père à l'adolescence, sans parents, proscrit de sa patrie, fut non seulement dépouillé par les membres de sa famille de tous les biens paternels, mais exclu aussi de leur intime familiarité et repoussé à un tel degré qu'il devait mendier auprès d'étrangers. Le sort du jeune homme était donc dur pour ces raisons, mais d'autant plus dur que les membres de sa famille, sans aucun sens de leur devoir, empêchaient tant qu'ils le pouvaient le jeune Philoponius de poursuivre les études des lettres, dans lesquelles il progressait en suscitant de grandes attentes chez les citoyens et l'admiration chez tous les lettrés. »

16. Fubini, Riccardo, Menci Gallorini, Anna, L'autobiografia di L.B.A., op. cit., p. 70 : Dedit enim operam iuri pontificio iurique civili annos aliquot, idque tantis vigiliis tantaque assiduitate, ut ex labore studii in gravem corporis valetudinem incideret [...] ex medicorum iussu studia haec, quibus memoria plurimum fatigaretur, prope efflorescens intermisit. Verum, quod sine litteris esse non posset, annos natus quatuor et viginti ad phisicam se atque mathematicas artes contulit; eas enim satis se posse colere non diffidebat, siquidem in his ingenium magis quam memoriam exercendam intelligeret. ("En effet, il s'adonna pendant quelques années au droit canon et au droit civil, en y dépensant tant d'efforts dans ses veilles, qu'à cause de ce travail assidu il tomba gravement malade $[\ldots]$ sous l'ordre des médecins il interrompit, au moment où sa jeunesse s'épanouissait, ces études qui fatiguaient particulièrement la mémoire. Mais, puisqu'il ne pouvait pas se passer des études, à l'âge de vingt-quatre ans il se consacra à la physique et aux mathématiques : il ne doutait pas qu'il pourrait les cultiver de façon satisfaisante, puisqu'il comprenait que dans ces disciplines il devait se servir davantage de l'intelligence que de la mémoire.») 


\section{DONATELLA BISCONTI}

militaire $^{17}$ : il s'agit, en somme, de ne pas sacrifier l'inclinaison naturelle, même lorsque celle-ci ne destinera pas l'enfant à suivre la profession marchande du père. La réussite exceptionnelle de Philoponius dans les études littéraires, dont toute la cité parle avec admiration, malgré les obstacles rencontrés au sein de la famille, est une démonstration de la volonté de progression, d'ascendance stoïcienne, marquée par le verbe "proficio " qui revient maintes fois dans les Epistulae ad Lucilium comme l'étoile polaire qui doit orienter notre travail quotidien ${ }^{18}$. Philoponius apparaît donc comme un héros face aux adversités : il affirme sa supériorité intellectuelle, qui n'est pas suffoquée mais aiguisée par l'exclusion de sa patrie et de sa famille. Il est cependant un héros persécuté qui se révolte contre son destin et maudit tous les pupilles dans une longue tirade parsemée d'exempla issus de l'histoire antique : autant d'hyperboles au sujet de ce que Philoponius n'a pas demandé aux dieux, richesses, pouvoir, gloire et ainsi de suite, qu'il prononce dans le délire de la maladie qui l'a frappé et dont ses proches ne se soucient pas. L'excès de la declamatio se place sous l'emprise de "furor " et d' "iracundia ${ }^{19}$ ", deux passions analysées dans le De ira et les tragédies de Sénèque, qui détruisent celui dont elles s'emparent. Si le Philoponius de Pupillus a une place dans la commisération des étrangers, il est par ailleurs exclu à jamais du bonheur. La révolte irrationnelle contre l'exclusion est alors un chemin sans issue, surtout si on lit Pupillus en liaison avec l'Intercenalis qui suit, Patientia, où les destins individuels sont

17. Alberti, Leon Battista, I libri della famiglia, in Opere volgari, op. cit., p. 43-44.

18. Cf. parmi les nombreux exemples, Seneca, Lucius Anneus, Epistulae morales ad Lucilium, livre III, XXIX, 3 : Sapientia ars est : certum petat, eligat profecturos, ab iis quos desperavit recedat, non tamen cito relinquat et in ipsa desperatione extrema remedia temptet. ("La sagesse est un art : qu'elle s'oriente vers ce qui est certain, choisisse ceux qui sont en mesure de progresser, s'éloigne de ceux dont elle désespère, et toutefois qu'elle ne les abandonne pas trop vite et tente même dans le désespoir les remèdes extrêmes. ") ; livre I, VI, 7 : "Quaeris" inquit "quid profecerim? amicus esse mihi coepi". Multum profecit: numquam erit solus. Scito esse hunc amicum omnibus. (" "Tu me demandes - dit-il - en quoi ai-je progressé? j’ai commencé à devenir l'ami de moi-même". Il a beaucoup progressé : il ne sera jamais seul. Apprends vite à être l'ami de tous. ")

19. Alberti, Leon Battista, Pupillus, in Intercenales, op. cit., livre I, p. 16: Quam rem dum pro virili ita ageret, mala tamen valitudo atque egestas in dies super vires urgebant, ut iam pro vetere proverbio in furorem verti patientia ceperit. Itaque tantis calamitatibus actus ac devictus adolescens, incenso animo indignatione et iracundia, hec in verba prorupit [...] ("Tandis qu'il supportait virilement sa situation, sa mauvaise santé et sa pauvreté jour après jour l'accablaient au-delà de ses forces, à tel point que, comme le dit l'ancien proverbe, sa patience commença à se tourner en fureur. Ainsi, le jeune homme, épuisé par tant de calamités, se laissant emporter par l'indignation et la colère, s'écria $[. .] »$.$) .$ 
ramenés à la « Necessitas " cosmique, qu’il faut accepter pour avoir droit à sa quote-part de bonheur.

Les péripéties de Philoponius se poursuivent dans Erumna, où la situation a changé. Le protagoniste reçoit la visite d'un ami qui s'étonne de le voir passer $\mathrm{du}$ rire aux pleurs en quelques secondes : Philoponius explique qu'il vient de s'entretenir avec un philosophe cynique, encore une fois l'un des masques de Niccoli, aux vêtements bizarres et sordides et aux attitudes théâtrales, qui avait poussé Philoponius à éclater de rire malgré son état d'esprit morose. Le philosophe croit ainsi l'avoir guéri juste avec quelques mots d'encouragement, puis disparait, en laissant son interlocuteur dans la même condition qu'avant. Rire et pleurs alternent de façon apparemment illogique : en fait, c'est le conflit entre la réalité extérieure, risible, si l'on y réfléchit bien, et le monde intérieur, demeurant opaque pour nos interlocuteurs, qui est représenté. L'ami qui survient ensuite ${ }^{20}$ remplace le philosophe cynique comme dans une scène de comédie, en faisant progresser la discussion et amenant Philoponius à reconnaître son erreur de perspective. Il incarne ainsi le rôle que les amis doivent jouer selon les mots de Consilium dans Anuli :

Verum habere hominem plures et oculos et aures fidissimorum diversas in partes intentos [sententia] affirmat, per quos queque ad se pertineat recognoscat. Neque id modo quod extra se sit, in quo aut consequendo aut vitando consilio et monitis iuvabitur, ope et opera levabitur; sed in primis in suorum iunctissimorumque verbis ac factis ipsum se quasi in speculo contemplabitur ${ }^{21}$.

20. Cf. Ponte, Giovanni, "La crisi della "Compagnia di corte”, l'Intercenale Erumna e il prologo alla Famiglia di L. B. Alberti ", in Tradizione classica e letteratura umanistica. Per Alessandro Perosa, a cura di R. Cardini, E. Garin, L. Cesarini Martinelli, G. Pascucci, Roma, Bulzoni, 1985, p. 159-171. Selon G. Ponte, l'ami de Philoponius qui tente de le réconforter, après le départ du philosophe cynique, pourrait être le cardinal Niccolò Albergati, qui fut en très bonnes relations avec les Alberti, et surtout, à Bologne, avec l'évêque Alberto di Cipriano Alberti, ce dernier protecteur de Battista, qui l'introduit comme interlocuteur dans Pontifex. G. Ponte date Erumna autour de 1436, à cause des allusions à la catastrophe financière des Alberti et de la présence d'Albergati, qui était rentré de Bâle à Bologne, où L. B. Alberti se trouvait à la suite d'Eugène IV, en mai 1436 (cf. p. 166-167). Erumna doit en tout cas être antérieure au 3 février 1437, date à laquelle Niccoli, présent sous le travestissement du philosophe cynique, disparut.

21. Alberti, Leon Battista, Anuli, in Intercenales, op. cit., livre XI, p. 778 : « [La maxime] affirme en fait qu'un homme doit avoir plusieurs yeux et oreilles, tournés dans toutes les directions, amis entièrement fiables pour connaître ce qui le concerne. Il sera soulagé non seulement pour ce qui est de la réalité extérieure, au sujet de laquelle il sera assisté par des conseils avertis dans ce qu'il faut suivre ou éviter; mais 


\section{DONATELLA BISCONTI}

L'ami d'Erumna remplit donc la fonction de double du personnage, et permet de surmonter le malheur par ses raisonnements paradoxaux, qui démontent l'excès d'émotion et le réduisent à ses termes logiques. Encore une fois, il est question de la situation de la famille Alberti, que nous retrouvons maintenant accablée par les querelles entre ses membres et la catastrophe financière. Ces malheurs retombent sur Philoponius, dépourvu du soutien des siens qui ne sont plus en mesure de lui procurer ni des amitiés influentes, ni une aide économique, sans compter la perte de prestige social.

[...] qui preterea splendore veteris familie nostre et claritate gloriabar - quas res a maioribus nostris comparatas patres nostri in exilio per alienas provincias errantes pulchre servassent - idem indignissima et detestabili contentione eorum, quibus in patriam restitutis regende tuendeque familie cura et officium commissum esset, omnem pristinam existimationem et amplitudinem domus nostre extinctam non sine lachrimis reminiscor ${ }^{22}$.

La solidarité familiale, inébranlable pendant l'exil, s'est désintégrée juste au moment où elle aurait dû se renforcer, lors du retour à Florence. Alberti généralise à nouveau le comportement de ses proches, dont il accentue les répercussions sur Philoponius à des fins pédagogiques. L'ami lui apprendra, au fil du raisonnement, à compter sur ses propres forces et à apprécier les qualités dont il est doué, si bien que Philoponius pourra conclure :

Itaque sic statuo prudentis esse, se velle eum esse qui $\operatorname{sit}^{23}$.

La formule, employée également ailleurs ${ }^{24}$, d'un côté met l'accent sur la nature de chacun, de l'autre, sur la volonté. Faire coïncider sa propre nature

surtout il se contemplera lui-même comme dans un miroir dans les faits et les mots des personnes les plus proches".

22. Alberti, Leon Battista, Erumna, in Intercenales, op. cit., livre IV, p. 306 : «[...] moi qui auparavant étais fier de la splendeur et de la renommée de notre ancienne famille - un héritage acquis par nos ancêtres que nos pères errants pendant l'exil dans des provinces étrangères avaient excellemment sauvegardé -, je ne puis évoquer sans pleurer l'estime et la puissance d'antan, désormais éteintes à cause de la querelle odieuse et détestable de ceux auxquels, une fois l'exil révoqué, était confié le soin de gouverner la famille et veiller sur elle".

23. Ibid., p. 320 : «Partant, j'établis que c'est le propre du prudent que de vouloir être celui qu'il est"

24. Alberti, Leon Battista, Templum, in Intercenales, op. cit., livre X, p. 650 : Atque inter deplorandum admonere singulos non desinebant: insanire illum qui nolit eum sese esse 
avec ses objectifs, voilà l'effort considérable que chacun doit poursuivre : il faut savoir accepter son sort dans le cadre d'une nécessité universelle, découvrir ce qu'on est et le réaliser. Pour ce faire, l'exil se révèle une condition importante, voire nécessaire, qui se déroule en trois étapes. Dans Erumna, l'exil apparait comme plus propice au maintien de la réputation et du patrimoine de la famille. En même temps, l'ami de Philoponius l'invite à prendre ses distances d'une famille qui ne sait pas conserver et transmettre ses valeurs aux jeunes rejetons et à ne pas confondre les malheurs des autres avec le sien pour en accentuer la gravité.

Neque id abs te non honeste et sancte desiderari dico, ut tua in familia adolescentes cura maiorum probatissimi et ab omni vitiorum labe intacti et nullis sordibus contaminati excrescant : tametsi etate preditum fore prudentia arbitrabar, ut recte intelligeres quam hi qui ab aliis partum sibi decus et lume servasse non didicerit, non per eos facile reliquam iuventutem ad glorie splendorem posse adduci. Sed non te audiam, si hac tua in expostulatione aliorum incommoda congeris, ut miseriarum tantum cumulum exaggeris. [...] Qua de re illos affines tuos missos faciamus a tuaque illos causa disiungamus ${ }^{25}$.

La deuxième étape, concomitante à la première, est présentée dans Divitie, où l'exil permet à Benedetto Alberti, le grand-père de Battista, de se détacher

qui sit, prudentisque officium profecto fore, quemcumque sors dederit locum, non odisse; ac veterem quidem consuetudinem, etsi incommodam et iniquam, potius ferendam quam novis institutis ipsum te atque alios in grave aliquod damnum ultimumque fortassis malum precipitem dandum. (" [Les pierres du temple] parmi les soupirs ne cessaient pas d'admonester chaque personne : il est fou celui qui ne veut pas être celui qu'il est, c'est le devoir du prudent de ne pas détester la place qui nous est réservée par le sort, quelle qu'elle soit; et il vaut mieux supporter l'ordre ancien, même s'il est inconfortable et injuste, que par de nouvelles institutions porter un préjudice grave à soi-même et aux autres et causer peut-être un mal irrémédiable. »)

25. Alberti, Leon Battista, Erumna, in Intercenales, op. cit., livre IV, p. 308, 310 : " Je ne dis pas que ton désir que les jeunes de ta famille sous le guide des anciens grandissent parfaitement vertueux, exempts de tout vice et de toute tache, ne soit pas honnête et juste : toutefois, je te croyais assez mûr et prudent pour comprendre correctement qu'il n'est pas facile que ceux qui n'ont pas appris à conserver pour eux-mêmes l'honneur et le prestige que d'autres leur ont procurés puissent adresser d'autres jeunes sur le chemin d'une gloire splendide. Mais je ne t'écouterai pas, si dans ton réquisitoire tu cumules les échecs des autres pour augmenter le cumul de tes malheurs. [...] Laissons donc tomber les membres de ta famille et distinguons ton cas du leur. » 


\section{DONATELLA BISCONTI}

de ses biens matériels et atteindre les vraies valeurs. Divitie conjugue l'attitude personnelle avec l'image publique du personnage, citoyen exemplaire, injustement exilé, mais fidèle à sa patrie et toujours prêt à la servir. Benedetto, qui exprime ses dernières volontés sur son lit de mort, est à mettre en parallèle avec Lorenzo mourant, entouré de ses enfants et de ses proches, dans le De familia. Il a pourtant dépassé la dimension des relations strictement familiales : entouré par ses amis, il refuse de léguer ses biens matériels, dont il ne reconnaît plus la propriété personnelle, et leur transmet un testament spirituel de valeur universelle. Ici l'éloignement, toujours géographique et physique, conduit le protagoniste à mettre en perspective sa propre vie et à hiérarchiser les biens. De la même façon, dans le Theogenius, Teogenio et son double Genipatro ressentent le besoin de mettre une certaine distance entre eux et la cité pour comprendre quels sont les biens véritables. En même temps, dans le Theogenius, une autre conséquence de l'éloignement apparait, l'inspiration littéraire : Teogenio écrit, dans un cadre idyllique, ses réflexions sur les troubles qui agitent l'État ${ }^{26}$. La campagne comme source d'inspiration est également présente dans la dédicace du livre VII des Intercenales à Pierre de Médicis. Dans cette lettre, ainsi que dans le Theogenius et dans le Profugiorum ab erumna - où les protagonistes rêvent de pouvoir s'éloigner de Florence pour avoir le loisir de se consacrer aux lettres et à la philosophie ${ }^{27}$-, l'éloignement ne peut être que temporaire : il permet de se ressourcer, mais condamne en même temps l'intellectuel à l'isolement et finit par s'avérer insuffisant. Ainsi, l'apologue du buffle et de la chèvre, dans laquelle Alberti se projette, dans le prologue du livre IV des Intercenales, dédié à Poggio, justifie le choix difficile de la chèvre, les herbes rares et amères, en d'autres termes, une littérature critique et autocritique, mais ce choix ne suscite que l'incompréhension du buffle ${ }^{28}$. L'autre aspect, indissociable du premier, est la dimension publique. Si l'exil des pères, les conséquences des déboires de la famille Alberti et la position de Battista en son sein sont subis comme des conditions non modifiables sinon par un élan volontariste et un appel à la rationalité, l'attitude de l'auteur vis-à-vis de la culture et de la politique florentines est en revanche un choix conscient.

26. Alberti, Leon Battista, Theogenius, in Opere volgari, a cura di Cecil Grayson, vol. II, Rime e trattati morali, Bari, Laterza, 1966, livre I, p. 58-59.

27. Alberti, Leon Battista, Profugiorum ab erumna libri III, in Opere volgari, a cura di Cecil Grayson, vol. II, Rime e trattati morali, Bari, Laterza, 1966, livre II, p. 138.

28. Alberti, Leon Battista, Prohemium ad Poggium, in Intercenales, op. cit., livre IV, p. 222. 
Tout au long des Intercenales, Alberti polémique avec les humanistes florentins, notamment avec Leonardo Bruni. Tout en revendiquant son originalité d'écrivain "facetus ", et en affirmant que le but du recueil est de non [...] studiosos instructiores, sed alacriores reddere ${ }^{29}$ (" rendre les savants non pas plus instruits, mais plus joyeux »), il vise tour à tour l'improductivité de la culture florentine, son éloquence vide et son imitation servile des modèles antiques, son culte de l'histoire en fonction de la reconstruction de l'image historique de Florence, et plus en général tout l'humanisme florentin (celui, du moins, que l'on désigne comme umanesimo civile), au nom d'une conception non pas "désengagée " mais «désintéressée " de la littérature : celle qui est également au centre de l'analyse du De commodis litterarum atque incommodis, où la marchandisation des professions libérales fait l'objet d'une attaque virulente. En contradiction apparente avec cette critique, Alberti, en quête d'intégration au sein des cercles culturels florentins, s'engage dans l'initiative du Certame coronario, qui aboutira, comme on le sait, à un échec cuisant. En réalité, l'initiative d'Alberti visait à promouvoir la littérature en langue vulgaire, et donc une idée de circulation culturelle qui n'était partagée ni par les humanistes florentins ni par l'aristocratie marchande florentine, qu'Alberti accuse d'être davantage intéressée par l'argent que séduite par les lettres.

Parallèlement, dans les Intercenales, Alberti ne cesse d'analyser implacablement les failles du système républicain florentin et en général des communes de son époque. Gallus, Bubo, Hostis sont autant de pièces où les comportements des citoyens, apparemment soumis à une logique rigoureuse, révèlent, par le biais du traitement humoristique, la violence des relations internes à la cité, l'hypocrisie des choix politiques, la subordination de la loi aux intérêts de groupes particuliers.

La lecture horizontale des Intercenales nous confirme cette impression d'étrangeté et d'isolement que les doubles d'Alberti ressentent vis-à-vis d'intellectuels vaniteux et de citoyens arrogants. Un des éléments les plus frappants est l'insistance sur le manque de reconnaissance pour le travail de l'homme de lettres qui ponctue les prologues et un nombre considérable d'intermèdes, comme Somnium, Cynicus (livre IV), et surtout Anuli (livre XI).

C'est cette dernière Intercenalis qui parachève l'œuvre. Si la distribution des intermèdes a un sens, et certes elle l'a, la position de Anuli à la fin du recueil marque un aboutissement. Philoponius, qui a consacré son existence

29. Alberti, Leon Battista, Prohemium (ad Leonardum Aretinum?), in Intercenales, op. cit., livre VII, p. 450. 


\section{DONATELLA BISCONTI}

à Minerve, non pas une Muse parmi d'autres, mais la déesse même du savoir, ou, pour mieux dire, des savoirs et de la sagesse, désespère pourtant de trouver une reconnaissance quelconque de son travail (représenté par les bagues qu'il a confectionnées sur ordre d'Espoir et de Conseil), en quelque lieu que ce soit :

Dum vagor foroque theatroque curiaque compitisque ac demum tabernis quoque lustrandis, en advesperascit. Nulli coemere [anulos], pauci spectarunt, omnes venale istiusmodi esse aurum negant ${ }^{30}$.

Inutile d'arpenter la cité pour trouver des amateurs de son œuvre. Philoponius ne veut plus entendre ni Consilium ni Genius ni Minerva ni Spes (encore une fois, des projections de sa conscience), mais finit par se laisser persuader par Fiducia d'entrer dans la Basilique :

Appulimus ad Basilicam. Ingrediamur. Heus, o! Ecquis hic est? Suntne hic studiosi? Suntne docti? At esse quos velim intelligo : video peritorum opera. Ingrediamur. Docti doctos servabunt, si erunt docti ${ }^{31}$.

Alberti choisit consciemment de renoncer à trouver sa place dans la cité à l'avantage d'une institution universelle, l'Église, où l'intellectuel s'exile pour

30. Alberti, Leon Battista, Anuli, in Intercenales, op. cit., livre XI, p. 770 : «Tandis que j'erre dans la place, le théâtre, l'assemblée citadine et les carrefours, et même dans les tavernes, voilà que le soleil se couche. Aucun acheteur des bagues à l'horizon, un petit nombre jette un coup d'œil, tous s'accordent à dire qu' un or de cette sorte n'est pas vendable".

31. Ibid., p. 789 : « Nous sommes arrivés à la Basilique. Entrons! Hé! Qui est là? Y a t-il des savants? Y a t-il des doctes? Je me rends compte qu'il y a ceux que je cherche; je vois l'œuvre d'auteurs compétents. Entrons! Les doctes sauveront les doctes, s'ils sont doctes ». La dernière phrase de la citation renvoie à la même expression dans le prologue du livre II, dédié à Leonardo Bruni, où, par l'évocation de Sostratus (en réalité Philostratus) et du philosophe Arrius, Alberti fait allusion à la traduction par Bruni de la Vita Antonii de Plutarque : apparemment un hommage à l'érudition du chancelier, en réalité, comme l'a démontré David Marsh, une transposition de la situation dont Philostrate et Arrius sont les protagonistes dans la Vita Antonii, par laquelle Alberti demande (avec une pointe d'ironie) au prince des lettrés d'être inclus dans son cercle grâce à un acte de magnanimité similaire à celui par lequel le "princeps» Auguste réintégra Philostrate parmi les doctes d'Alexandrie. Cf. Marsh, David, "Textual problems in Intercenales", Albertiana, II, 1999, p. 125135 , notamment p. 134-135. 
être sauvé par d'autres intellectuels ${ }^{32}$. Ni refuge, ni port du salut, la «Basilica » est le lieu où la virtus laïque et terrestre albertienne, qu'il définit dans les mêmes années dans le De iure ${ }^{33}$, déploiera enfin son excellence, mais connaîtra également d'autres exclusions et d'autres exils sur lesquels planera le rire amer et paradoxal du Momus.

\section{Donatella Bisconti}

32. Il est à remarquer qu'Alberti emploie le mot "Basilica " pour désigner la curie romaine, tandis que Lapo da Castiglionchio intitule De curiae commodis son dialogue, écrit en 1437 - donc dans les mêmes années où Alberti composait les Intercenales -, qui illustre les avantages de la vie à la curie. "Basilica » exploite l'ambiguïté du mot, renvoyant à la fois au lieu où les Romains administraient la justice, et à un édifice du culte chrétien (qui d'ailleurs à l'origine était une " basilica » romaine, reconvertie à une fonction religieuse à partir de Constantin) : en effet, Alberti n'indique qu'une motivation laïque, l'échange entre intellectuels, pour justifier son choix de tourner le dos à la cité. Lapo da Castiglionchio, un intellectuel en quête tout au long de sa vie d'un emploi stable, de préférence dans le milieu de la curie, hiérarchise en revanche ses motivations : tout d'abord, la curie est le milieu le plus adapté pour atteindre le bonheur dans l'au-delà ; ensuite, dans la curie on peut cultiver toutes les vertus et notamment les vertus cardinales, grâce aux relations avec des hommes compétents et experts dans la politique et la diplomatie; enfin, la curie est le lieu où se trouve la plus grande concentration de doctes et où l'on peut se consacrer paisiblement aux études. Cf. Lapo da Castiglionchio, Dialogus de curiae commodis, in Prosatori latini del Quattrocento, a cura di E. Garin, Milano-Napoli, Ricciardi, 1962, p. 169-211, notamment p. 183-210.

33. Alberti, Leon Battista, De iure, publié par Cecil Grayson, "Il De iure di Leon Battista Alberti ", in Tradizione classica e letteratura umanistica. Per Alessandro Perosa, a cura di R. Cardini, E. Garin, L. Cesarini Martinelli, G. Pascucci, Roma, Bulzoni, 1985, p. 173-194: Virtutem hoc loco ex hominum more appellamus, ut eum in quo aut manus aut lingua aut vox aut pes aut quidvis istiusmodi doctrina aliqua excultum sit, et qui fidibus, qui pictura, qui istiusmodi aliqua arte sit edoctus, virtute illum ornatum dicamus. Tum etiam et maxime virtute excellere eum dicimus, in quo litterarum ac rerum difficillimarum ratio et cognitio adsit. ("Ici nous définissons la vertu selon l'usage des hommes, d'après lequel nous disons qu'un homme est orné de vertu lorsque ses mains ou sa langue ou sa voix ou ses pieds ou une partie quelconque se parent de la pratique de quelque savoir, et qu'il a des compétences dans les instruments de musique, la peinture ou un art de cette sorte. Mais surtout nous disons que celui qui a une cognition fondée en raison des lettres et des choses les plus difficiles excelle dans la vertu »). 